Chapter 7

\title{
Five Star Crisis Management - Examples of Best Practice from the Hotel Industry
}

\author{
Outi Niininen \\ Additional information is available at the end of the chapter \\ http://dx.doi.org/10.5772/55209
}

\section{Introduction}

The background for this study is in the complex and fragmented business environment of today when many organisations are interdependent from each other across the globe thus making it harder for companies to insulate themselves against a crisis event [1]. Furthermore, the media of today is ready to inform us of any critical events around the world at a moment's notice [2, 3]. The 'everyone can be a journalist' culture is encouraged by popular media requesting photographs and video footage from individuals currently caught in an unexpected chain of events. Today, words like 'crisis' and 'disaster' are featured in everyday vocabulary to grab attention, thus resulting in a depreciation of the actual meaning of this term. Overall, an atmosphere hungry for crisis has been created.

At the same time, there has been a plethora of international crisis demonstrating how fragile the business environment can be. The most cited crises have been caused by nature (e.g. tsunami, hurricanes, bushfires, floods or disease) or by man (e.g. terrorism and the current Economic Tsunami). In this environment, the Hotel Managers' duty of care should also involve planning and preparing for unforeseen events; running 'what if' scenarios, designing action plans for all departments, allocating individual responsibilities; building back-up capacity and training their staff to respond in appropriate manner to security concerns. Therefore, the aim of this paper is to highlight good procedures already practiced by hotels participating in this study. The study Methodology consisted of in-depth interviews with Hotel Managers or Hotel Security Managers located in Hong Kong, London and Finland. Many of these hotels were associated with large multinational chains and the most of the interviewees had managed a variety of crises situation, often in contrasting cultural or geographical locations. 
This paper is set out in the following structure: the basic crisis management concepts are outlined and applied to the tourism (and hospitality) settings. This is followed by a brief outline of the methodology utilised. The findings/discussion section is structured to reflect the key practical tips offered by the interviewees: 'Being prepared for crisis situations'; 'Managing costs during crisis'; 'Make full use of local advice'; 'Maintaining good communication with guests' and how to 'Use technology to enhance hotel security'.

\section{Crisis management}

Crisis management has its roots in strategic planning incorporating contingencies for unexpected events. The challenge for organisations is to recognise the early 'warning signals' and take appropriate action. Crisis can be classified by several variables: those resulting from internal actions of the company vs. trends taking place in the market place or changes in the immediate environment outside the organisation. Another approach to crisis classification is: the crisis that can be managed vs. crisis that manage us [the organisation][3].

Generally speaking, crisis as an event is characterised by its unexpected nature inflicting severe impact on those involved. Moreover, a crisis typically demonstrates the characteristics of suddenness, uncertainty and time compression thus demanding immediate action from the manager - often the crisis management decision-making is also based on incomplete facts [4, 5]. How any of these challenges are managed can determine the magnitude of the impact a crisis will have on the organisation. Prompt and expertly dealings with a crisis situation and those affected by this crisis can also open new opportunities for future business success [6]. Unfortunately the crisis reported in the news today will inevitably result in some financial losses as well as human suffering. As a result some businesses will be closed or relocated. The cynics amongst us argue that the crisis simply brought forward what was inevitable and that a 'natural process of weeding out the weak' has taken place.

Regrettably crisis events are commonplace, [7] as organisations are regularly dealing with the pre-crisis; during-crisis or post-crisis issues thus highlighting the need for an early 'diagnosis and treatment' of a crisis to reduce the negative impact to the company. Moreover, a crisis can also be a chain of events that are impossible to control or plan for, thus resulting in 'death or significant injuries' to those involved, disrupt the business operations as well as damage to or destruction of company assets. In other words, a crisis situation turns 'business as normal' into impossibility [3, 6].

Tourism as an industry has many characteristics that can make a crisis more probable, magnify the impacts of a crisis and attract extreme media coverage for the event. Firstly, tourism is big business and often cited as the 'largest peacetime industry' [8] and tourism is also advocated as the industry offering sustainable (and quick yielding) development opportunities to the least developed countries. Secondly, the tourism industry is highly integrated with several other industries. In other words, the ripples of a crisis event from the hotels' supply chain can result in a flood of issues/problems for the business of providing hospitality [1]. Thirdly, the tourism industry is about the movement of people 
(i.e. all individuals with the means and motive to travel are potential tourists). Thus the variety of psychological or social responses to unexpected events from the guests of an international hotel will challenge even the 'best laid [crisis] plans' as well as the communications skills of experienced PR Managers to maintain calm [2]. Fourthly, since the consumption of a tourism product requires the customer to travel to a destination the demand for tourism products is sensitive to reports on security and health issues; and finally, many tourists are attracted to fragile locales particularly vulnerable to the forces of nature (e.g. tropical weather, proximity to sea and even seismic activity) or destination with low degree of infrastructure development (so called 'unspoilt' destinations) [5, 9-11].

Moreover, tourism participation is a discretionary activity for most international travelers, many countries (or destinations) have invested heavily on new campaigns in order to acquire the misplaced inbound tourists or to generate alternative demand from domestic travelers [12, 13]. Therefore, in tourism industry, '... it is no longer a question whether [a crisis] will arise, but when and how it will be dealt with' [7] therefore hotels' crisis plans should be more generic in nature, thus offering personnel accepted behavioural protocols without attempting to script for every eventuality. The wide variety of potential crisis in the hospitality industry also supports this argument.

\section{Methodology}

The findings in this paper are based on in-depth interviews conducted during October 2008. Non-probability sampling was used to select locations due to the range of past crisis events varying from financial crisis (e.g. current financial crisis worldwide and the financial crisis in Finland in the early nineties), health concerns (e.g. SARS reports linked to Hong Kong) to terrorism (e.g. the UK and London) reported in those locations. At each city, major stakeholders from educational institutions to trade associations were approached to request contact details for hotel managers willing to participate in research projects. Individual interview requests were made through e-mail and the final number of interviews conducted was 12 (three in Hong Kong; six in London and three in Helsinki).

Each respondent received the planned interview questions in advance together with background information for this study and the University research ethics procedures. Although General Managers were approached initially, in some cases the interview request was delegated to the Head of Hotel Security thus resulting in a mixture of nine Hotel Managers and three Hotel Security experts. Collectively, the respondents hold decades of crisis management experience across the world with focus on the hospitality industry as well as other segments of the security industry like Police or Fire departments. Naturally, the personal crisis experience of each person influenced the examples and policies highlighted by each individual thus making the data more rich. The 'default' identification for each respondent was to code them by location e.g. 'Hotel A, HK' or 'Hotel K, UK' etc. 


\section{Findings and discussion}

The first observation during the data collection process was how well prepared all hotels interviewed were. Most interviewees arrived with their crisis management plans, in one incident; a major evacuation practice was due to take place later on the same day.

The probability of incidents or crisis events taking place in a hotel increases with the volume of business and the size of the establishment; 'when the company [the hotel or the chain of hotels] provides thousands of bed nights every year, something is likely to happen'. One important contributor to hospitality industry incidents or crises are the guests or visitors to the property. The Hotel Manager can select their staff members and train them to respond to events in the desired way. Unfortunately, such luxury does not always exist with the visitors and guests. This is not to say that the visitors would be deliberately causing harm but the mixture of varying cultural norms, language barriers and lack of understanding of the prevailing condition can confuse the guests, thus causing an incident or making the crisis control extremely difficult for the hotel employees [2]. However, clever use of the extended Marketing Mix resulting in the market positioning, the rates charged as well as the physical evidence of the hotel can, on one hand, discourage segments that might not respond well to the guidance by the hotel employees. On the other hand, the physical evidence evident in a $5^{*}$ hotel can also have a surreal calming effect on visitors not used to such luxury and formality possibly resulting in the incidental visitor to 'be on their best behaviour'. Furthermore, policies like 'no Buck's nights' as well as good records of past visitors can also be used to discourage segments outside the hotel's target market.

The reported crises experienced by the respondents can be classified as internal (within their property) and external (outside their property). The internal crises varied from technical/ power failures to death of a hotel guest and the external crises varied from mass cancellation of hotel bookings due to the current financial crisis, to accidental or deliberate incidents of vehicles crashing into the hotel buildings (such deliberate damage to the hotel property and risking of human lives would be classified as terrorism) (Hotel A, HK).

All respondents referred to the Hotel Manager's responsibility for the well being and safety of staff and guests. In fact the Hotel Manager's duty of care was the most commonly cited expression across all interviews. Another conclusion from the data collected is that hotel security was often delegated to individuals who already had responsibility for Occupational Health and Safety (OHS). Furthermore, basic hotel security training was also cited to be part of the overall induction to new staff members (Hotel L, UK).

\subsection{Being prepared for crisis situations}

All respondents advocated the importance of being prepared for crisis situations and most respondents arrived to the interview with their Crisis Preparation Manuals. Furthermore, in one hotel, a crisis response practice was scheduled to take place shortly after the interview. The obvious/visual display of security measures (e.g. metal detectors at the entrance) is appropriate for specific target segments or situations only. The examples illustrated the varied 
degree of security measures needed for mega events like the 2012 London Olympics (all London respondents) and offering hotel services to the top politicians in the EU or other VIP guests (Hotel E, Finland). In such instances the security protocols are set externally and specialist security personnel is provided by the event organisers. A more common request for additional security takes place when a corporate client uses the hotels conference facilities for a strategically significant meeting. In these situations public access to some parts of the hotel may be restricted (Hotel D, Finland).

The day-to-day security operations included the 'secondary' security role assigned to most front line employees. This approach allows for a more discrete security operations and maintaining the appropriate quality of service. For example, the concierge limits the access to the hotel by suspicious individuals (Hotel D, Finland and Hotel L, UK) and front of house personnel diplomatically offering assistance to visitors who do not seem to belong. Such helpful attention to visitors was also experienced by me as the 'out of place interviewer'.

\subsection{Managing costs during crisis}

Managing costs effectively during any crisis is probably the key to being able to remain in business during and after crisis, as any reduction in hotel occupancy rates will translate into a drop in the revenues. However, this cost saving exercise should be conducted in a way that allows the hotel to bounce back quickly once the demand for their services returns. For example, short term cost cutting can result in years of Good Will from all stakeholders disappearing, thus resulting in difficulty in securing supplies and recruiting personnel after the crisis is over. A key cost area for service industries is the payroll, therefore if substantial cost cutting is required to survive the current crisis. Therefore, the Hotel Manager will need to 'look for ways of reducing costs whilst maintaining their support to the staff' (Hotel C, HK). The labour cost and capacity can be reduced by asking volunteers to take vacation during crisis (Hotel B, HK) or by changing operating procedures (Hotel C, HK). Another response to a crisis event is to stop recruiting (Hotel G, UK).

Moreover, a significant drop in demand and revenues due to a crisis will also require cutting costs in any area possible. For example, the supply costs for power and/or electricity can be managed by closing areas of the property; e.g. by closing a floor and concentrating the guests accommodation in dedicated areas will save power and allow for better utilisation of staff (Hotel B, HK). By concentrating the fewer visitors to specific areas can also help create the perception that the hotel is not badly hit by the current crisis situation, e.g. the worldwide economic crisis. And finally, a drop in demand and revenues should also prompt the Hotel Management to re-evaluate further investment plans. In conclusion, during a crisis event it is important not to waste resources (Hotel G, UK).

\subsection{Make full use of local advice}

The advice to 'make use of local advice' that is often also offered for free, was most frequently communicated by interviewees based in Finland and London. However, such observation 
could be purely incidental; e.g. in Finland only three respondents were interviewed, one of them had recent experience in providing accommodation for high ranking EU delegates (Hotel E, Finland); the London hotels were getting ready for the 2012 Olympics, and therefore, a greater collaboration between hotels and authorities is to be expected.

The type of free advice offered by local authorities (as well as other organisations) is determined by the location of the hotel. For example, the location of the hotel will determine the required building standards as well as the requirement for a formal rescue plan to be lodged with the authorities, the frequency of relevant safety inspections, and the extent to which the authorities are working with the hotels in a proactive manner (Hotel D, Finland).

Two of the London Interviewees made unprompted comments on how they 'welcomed the need for fire compliancy and regular inspections' since 'the fire certificate ensures compliance with fire safety'. Moreover, through a thorough crisis preparedness plan the hotels were also able to save in their insurance premiums (Hotel G, UK). Furthermore, 'regular fire inspections help hotels enhance/update their fire safety plans' (Hotel L, UK).

The closer links between the London Metropolitan Police and the hotels had also resulted in the Police issuing regular updates on specific criminal activities targeting hotels as well as fraudulent individuals to watch out for (Hotel L, UK). Finally, the time to develop positive relationships with authorities like the police or fire/rescue departments is during the quiet times, as good links to the authorities would be needed during crisis (e.g. Hotel L, UK; Hotel D, Finland).

\subsection{Maintaining good communication with guests}

In a large hotel good customer records are the basis for excellent customer relations. These records will allow the hotel to learn about their frequent guests and to keep their loyal customers (e.g. Hotel D in Finland). However, such inside knowledge should not be used to exhaust the guests with direct mail but rather preserve such communications for times when there is a need to attract business (Hotel L, UK). Furthermore, good guest history [and incident reports] also help dealing with complaints that may be received later.

Accurate record keeping should not be limited to the Customer Relations Department but be a standard across all the departments within the hotel. Moreover, all well documented procedures can also stop a minor incident from becoming a major crisis. For example, accurate temperature control in food preparation processes, maintenance work carried out, and even the details of deliveries taken, may be questioned months after the guest has departed. One Hong Kong based Hotel Manager (Hotel A) commented on guests from several months ago inquiring whether they had been served milk products sourced from China at any stage during their visit to the hotel. These guests had heard international news reports about contaminated baby milk in China and wanted to know if any food or beverages consumed in the hotel could have traces of such contaminated milk (for more on milk contamination in China please see [14] ). This example highlights the need of thorough record keeping across all departments within the hotel, as these files are needed to reply to a concerned guest whether they have been exposed to any external health risks 
during their stay at the hotel (Hotel A, HK). For example, international news reports on poor air quality, polluted rivers or even faulty air-conditioning units near the hotel location could cause some negative word-of-mouth if the hotel is not able to demonstrate appropriate duty of care through well maintained records.

Hotels also provide the venue and catering for different types of events. Such functions serve specialist menus to groups up to several hundred guests. The participants of these specialist events can continue their celebrations in various independent restaurants outside the hotel and may even conclude the night with a snack from a street vendor. A guest suffering from food poisoning might blame the hotel for their symptoms even when they are not able to identify all food items consumed during their celebrations. A hotel can eliminate false accusations of poor food hygiene by preserving/freezing a small sample of food prepared for a major banquet. If required, the hotel can get the preserved food samples analysed by an independent laboratory before accepting any liability (Hotel B, HK). Finally, the prevalence of food allergies today requires detailing and standardising the ingredients for every item on the menu (Hotel D, Finland).

\subsection{Use of technology to enhance hotel security}

Technology can be used to enhance the overall hotel security and cameras in corridors as well as other public spaces are the norm today (Hotel D, Finland). Well positioned cameras and other visible security measures are welcomed by the guests as they increase the perception of security (Hotel I, UK). However, excessive use of cameras can give the impression of specific security problems on the premises (Hotel L, UK). Electronic key cards are also the norm today and the CCTV is viewed invaluable in securing the hotel premises (Hotel K, UK).

Recording security cameras at key positions, alarms and motion sensors indicating unauthorised access, as well as digital monitoring of access to the premises, were nominated as common tools for maintaining hotel security. The hotels in Finland were already using external security agencies to monitor their alarms as well as patrol the premises (Hotel D). Some of the London based hotels were considering such an option as well (Hotel G).

The Internet and e-mail were seen as a tool for enhancing the hotel security as well as a potential risk to the operations or the image of the hotel. The need for up-to-date firewalls to protect the hotels' computer networks was highlighted by some of the interviewees (e.g. Hotel D, Finland). Furthermore, the need to backup the data together with alternative communications channels outside the primary premises was also identified (e.g. Hotel B, HK; Hotel, UK). Moreover, licence plate readers were installed in the garage of one busy London hotel (Hotel L, UK). Finally, the collaboration between hotels and the police also involved e-mail alerts of scams targeting hotels complete with photographs of the suspects the hotels should look out for (Hotel D in Finland; Hotel K, UK).

Finally, many types of technology was utilised by all interviewees in maintaining the hotel security, and to some extent overt security measures facilitated by technology (e.g. electronic key cards, security cameras in public places) are now expected by the hotel guests. However, 
the underlying guideline for the use of technology in hotels was the need to 'protect the privacy of our guest' (Hotels $\mathrm{H}$ and L, UK),

\section{Conclusions}

The main contribution from this study is the accumulation of practical advice that all Hotel Managers can utilize, regarding of the size of their hotel. The practical advice offered by the participants of this study is based on accumulation of decades hotel management experience across the globe. The findings from these interviews can be grouped under the following headings: Being prepared for crisis situations; Managing costs during crisis; Make full use of local advice; Maintaining good communication with guests; and the Use of technology to enhance hotel security. The need to offer high levels of service, local collaboration as well as the secondary security related responsibilities of all existing staff were another overlapping theme throughout the interviews.

The findings of this study could be presented as a benchmark for good practice for crisis prevention in large international hotels. The examples cited by the interviewees covered crisis situations from small accidents to terrorism or accidental death within the premises.

The limitations of this study are, therefore, outcome of the limited resources (time and funding for data collection) and the bias towards large, international hotels. The timing of the data collection also coincided with the Economic Tsunami of 2008 where the signs for economic slowdown were already evident (reduced advance bookings, lower than usual occupancy rations as well as the cancellations of large bookings).

The conclusion of this paper should also cite two quotes reflecting the many years of crisis management experience by the Interviewees: 'every cloud has a silver lining' (Hotel K, UK) and that hotels can 'come out of crisis stronger' (Hotel I, UK)

\section{Acknowledgements}

This study was funded by La Trobe University Outside Studies Programme

\section{Author details}

\section{Outi Niininen* $^{*}$}

Address all correspondence to: o.niininen@latrobe.edu.au

Senior Lecturer in Marketing, LaTrobe Business School, La Trobe University, Bundoora, Victoria, Australia 


\section{References}

[1] Corey, C. M, \& Deitch, E. A. Factors Affecting Business Recovery Immediately after Hurricane Katrina. Journal of Contingencies and Crisis Management, (2011). , 169-181.

[2] Wester, M. Fight, Flight or Freeze: Assumed Reactions of the Public During a Crisis. Journal of Contingencies and Crisis Management, (2011). p. no-no.

[3] Laufer, D. Charting a Course Through Crisis. BizEd (2010). September/ October): , 46-50.

[4] Stafford, G, \& Yu, L. and Kobina Armoo, A Crisis management and Recovery, How Washington, DE., Hotels Responded to Terrorism. Cornell Hotel and Restaurant Administration Quarterly, (2002). , 27-40.

[5] Irvine, W. a. A. A.R., The impacts of Foot and Mouth Disease on peripherial tourism area: The role and effect of crisis management. Journal of Travel and Tourism Marketing, (2005). , 47-60.

[6] Kash, T. J. a. D. J.R, Crisis management: prevention, diagnosis and intervention. Leadership and Organization Development Journal, (1998). , 179-186.

[7] Henderson, J. a. N. A., Responding to crisis: Severe Acute Respiratory Syndrome (SARS) and hotels in Singapore. International Journal of Tourism Research, (2004). , 411-419.

[8] Tarlow, P. (2006). Issues in health, safety and security. e-Review of Tourism Research 4.

[9] Mckercher, B. a. P. R., Privation as a stimulus to travel demand? Journal of Travel and Tourism Marketing, (2005). , 107-116.

[10] Méheux, K, \& Parker, E. Tourist sector perceptions of natural hazards in Vanuatu and the implications for a small island developing state. Tourism Management, (2006). , 69-85.

[11] Cioccio, L, \& Michael, E. J. Hazard or disaster: Tourism management for the inevitable in Northeast Victoria. Tourism Management, (2007). , 1-11.

[12] Fall, L. T. The increasing role of public relations as a crisis management function: An empirical examination of communication restrategizing efforts among destination organization managers in the wake of $11^{\text {th }}$ September 2001. Journal of Vacation Marketing, (2004). , 238-251.

[13] Stanbury, J, \& Pryer, M. and Roberts, A Heroes and Villains- Tour operator and media response to crisis: An exploration of press handling strategies by UK adventure tour operators. Current Issues in Tourism, (2005). , 27-40.

[14] AnonChina say milk contamination under control. (2008). ABC. 
